1 Artificial Metalloenzymes on the Verge of New-to-nature Metabolism

Authors: Markus Jeschek, ${ }^{1}$ Sven Panke, ${ }^{1}$ and Thomas R. Ward ${ }^{2}$

${ }^{1}$ Department of Biosystems Science and Engineering, ETH Zurich, Basel, Switzerland. ${ }^{2}$ Department of Chemistry, University of Basel, Basel, Switzerland.

${ }^{*}$ Correspondence: markus.jeschek@bsse.ethz.ch (M. Jeschek).

Keywords: artificial metalloenzymes, synthetic biology, biocatalysis, directed evolution

\title{
Abstract
}

Residing at the interface of chemistry and biotechnology, artificial metalloenzymes offer an attractive technology to combine the versatile reaction repertoire of transition metal catalysts with the exquisite catalytic features of enzymes. While earlier efforts in this field predominantly comprised studies in well-defined test-tube environments, a trend towards exploitation of artificial metalloenzymes in more complex environments has recently emerged. This includes the integration of these artificial biocatalysts in enzymatic cascades and reaches out to their utilization in whole cell biotransformations and in vivo, opening up entirely novel prospects for both preparative chemistry and synthetic biology. Here we highlight selected recent developments with a particular focus on challenges and opportunities for the in vivo application of artificial metalloenzymes.

$<$ Trends Box>

Artificial metalloenzymes (ArMs) are an emerging form of non-natural biocatalysts, which allow to create biocatalytic novelty with potential applications in preparative chemistry and synthetic biology.

Initial engineering efforts for ArM creation have been conducted in well-defined in vitro systems based on purified protein variants and therefore systematic directed evolution of ArMs as well as their introduction into cellular pathways has been hitherto largely limited.

More recently, a trend towards utilization of ArMs in whole-cell systems and in vivo has emerged, which is associated with a number of critical obstacles yet to be overcome. This transition shows great promise for the sustainable production of commodity chemicals and new-to-nature metabolites using ArMs.

$<$ Trends Box> 


\section{Artificial Metalloenzymes}

Artificial metalloenzymes (ArMs hereafter; see definition in Box 1) are a class of synthetic biocatalysts, which combine attractive features of enzymatic and transition metal catalysis. While enzymes are well-known for their exquisite catalytic performance comprising high reaction rates, turnover numbers (TONs) and selectivity as well as mild reaction conditions [1,2], they are limited to the arsenal of reactions that has emerged during natural evolution [3]. In contrast, transition metal catalysts offer a broad range of reaction mechanisms, many of which are not found amongst natural enzymes, providing a valuable toolbox for synthetic chemistry. However, homogeneous catalysts are often incompatible with natural enzymes and numerous cellular metabolites. Combining these two seemingly unrelated domains by creating ArMs, which catalyze new-to-nature reactions and, importantly, are genetically encoded and hence evolvable, offers great synergistic potential. This was first demonstrated by Wilson and Whitesides who, by incorporation into avidin, endowed a biotinylated rhodium catalyst with enantioselectivity for a hydrogenation reaction, while in the absence of the protein racemic product was formed [4]. The potential of ArMs has since been demonstrated for several protein scaffolds and target reactions. As this is the subject of several excellent reviews (e.g. [3, 5-9]) it shall not be comprehensively discussed here.

Previous work on ArMs predominantly focused on studies of reactions in defined in vitro systems relying on purified protein variants. Consequently, their genetic optimization was limited to few target residues. Screening of large numbers of genetic variants, however, bears great potential for enzyme development, which was recently demonstrated for a highly efficient artificial aldolase [10].

In parallel, a trend towards application of ArMs in more complex systems is prevailing, which includes employing them in cell-free extracts $[11,12]$, whole-cell biotransformations [12-16], and in vivo [17, 18], as well as their introduction into multienzyme reaction systems including regulation [19-23]. This transition towards integration of bioorthogonal chemistry into synthetic biological systems might drastically accelerate directed evolution of ArMs and by far exceeds their aforementioned potential for preparative chemistry [24, 25]. One can envision the use of ArMs in novel biochemical pathways to produce previously inaccessible compounds, which could contribute to the inevitable transition of our petroleum-based economy 


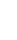

towards sustainable production. While this transition is arguably cumbersome, the field of ArMs is currently experiencing disruptive change and in vivo application seems well within reach. This review highlights important recent proceedings in the creation of novel reactivities using ArMs and emphasizes critical challenges and opportunities for their utilization in living cells.

$<$ Box 1>

\section{Definition of Artificial Metalloenzymes (ArMs)}

For the purpose of this review, an artificial metalloenzyme (ArM) shall be defined as a protein (>50 amino acid residues) which contains at least one metal ion playing a crucial role in catalysis and which can be regarded as "artificial" due to at least one of the following attributes:

- it contains a non-canonical catalytic metal (i.e. not found in natural enzymes)

- it catalyzes a non-natural reaction (-mechanism) (incl. repurposing of natural metalloenzymes!)

- its protein scaffold is designed de novo

ArMs are composed of two basic components, a protein part or "scaffold", which in its apo-form is catalytically inactive, and a metal component or "cofactor", which includes a metal ion or a complex thereof. The definition applied in this review excludes metalcontaining peptide catalysts, (<50 amino acid residues) which, while undoubtedly an important area of research, shall not be reviewed herein but have been discussed elsewhere [26, 27].

$<\mid$ Box 1> 


\section{ArMs for the Creation of Catalytic Novelty}

Engineering of enzymes for new-to-nature reactions bears great potential for industrial applications providing efficient and ecologically friendly solutions for synthetic chemistry [7]. Different approaches have been pursued to generate catalytic novelty using metalloenzymes, which can be roughly divided into i) repurposing of natural enzymes, ii) enzyme (re-)design, and iii) artificial cofactor approaches (Figure 1).
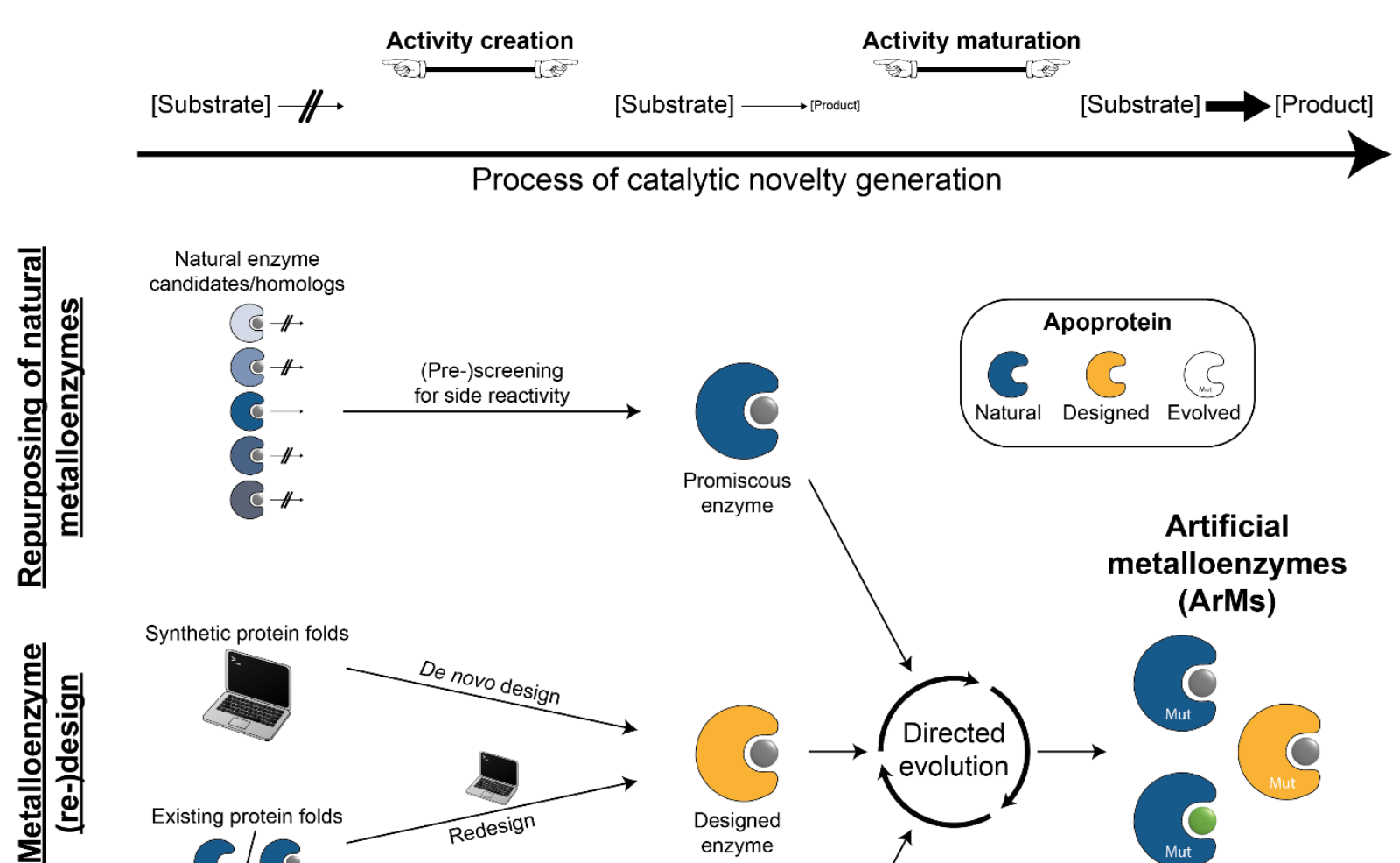

$$
\begin{aligned}
& \text { Natural enzyme } \\
& \text { candidates/homologs }
\end{aligned}
$$

की

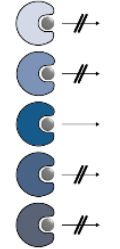
s
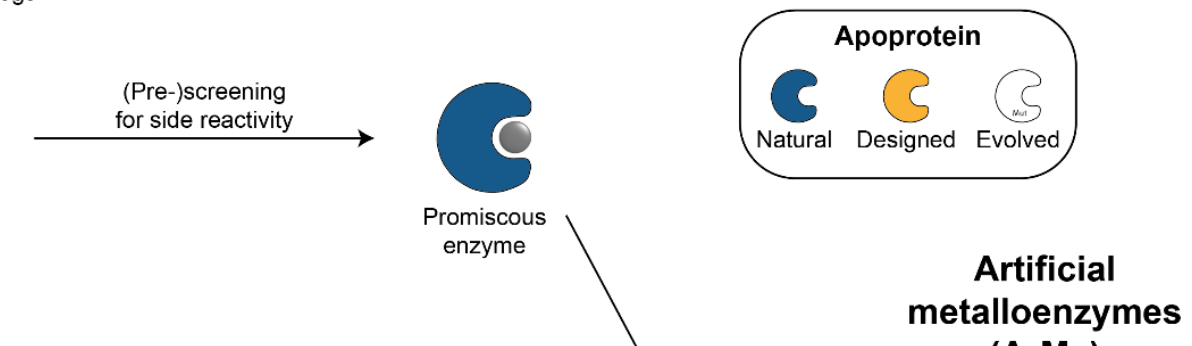

Synthetic protein folds
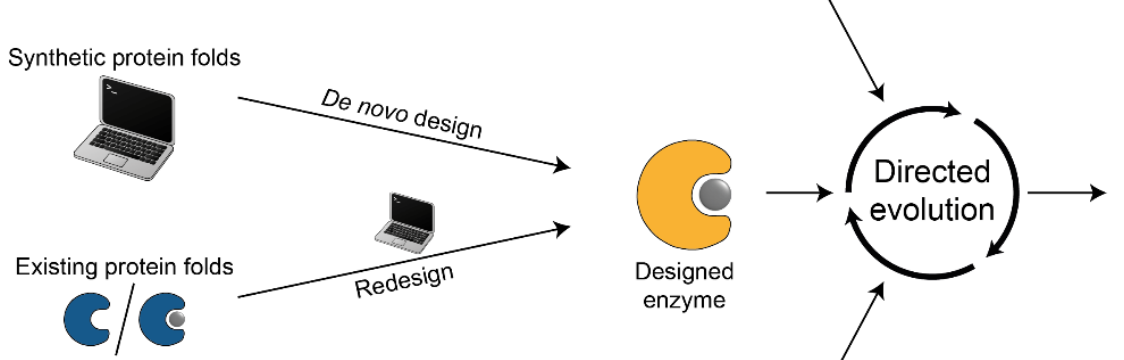

(ArMs)
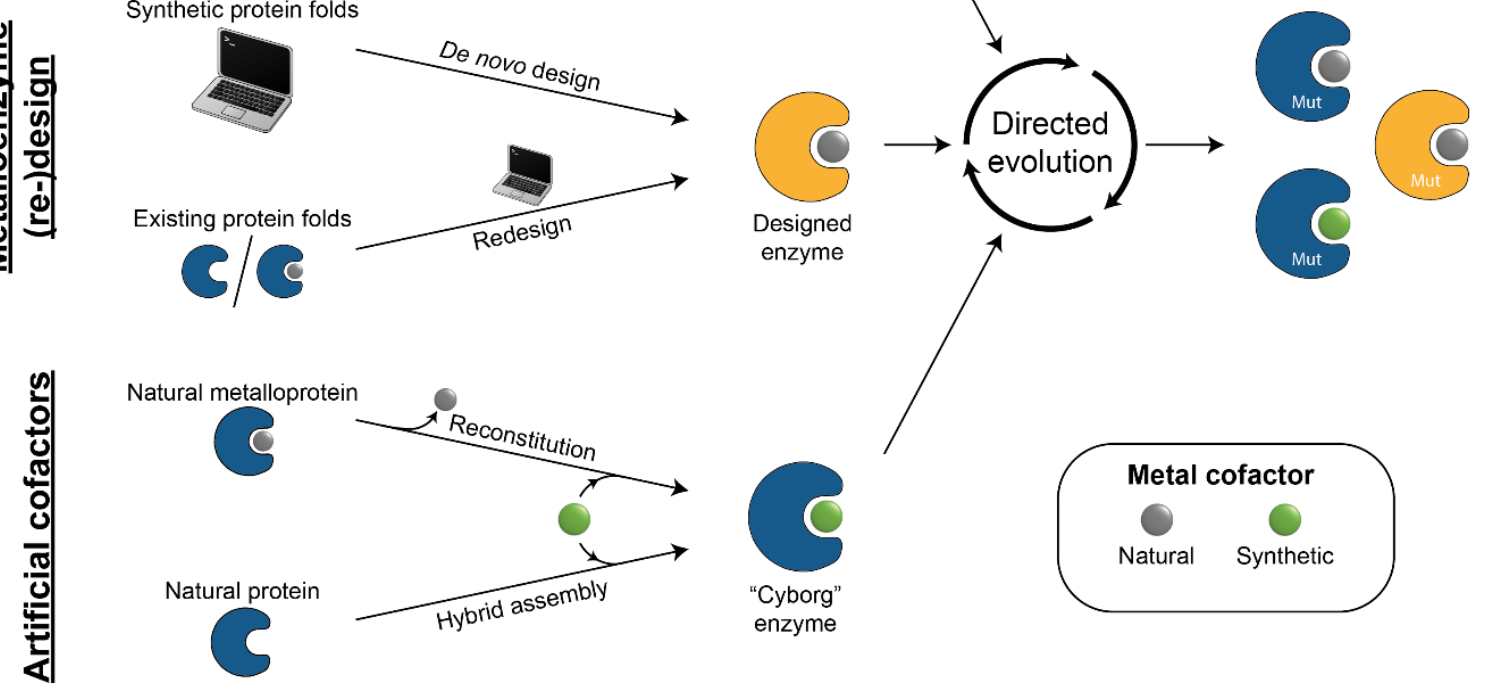

Figure 1. Strategies for the generation of emerging catalytic activity by creation of artificial metalloenzymes (ArMs). Natural metalloenzymes can be repurposed to catalyze entirely novel reactivities provided a promiscuous enzyme candidate can be identified which exhibits at least rudimentary side reactivity for the desired reaction. In the absence of the latter metalloenzymes with basic activity can be designed, either from scratch (de novo) or relying on existing proteins into which active metal centers can be introduced (redesign). Alternatively, artificial cofactors with intrinsic activity can be introduced to endow the cognate protein with activity. This can be achieved by reconstituting 
natural metalloenzymes with synthetic metal(-cofactors) or by introducing the latter into proteins without native metal-binding properties (e.g. by covalent or supramolecular anchoring). Once minimal activity is established by one of the aforementioned methods, directed evolution can be used to evolve the ArMs for the desired application (for a selection of recent studies applying the different strategies please refer to Table 1).

Repurposing relies on inherent promiscuous activity of natural enzymes and directed evolution of this feature to practically useful extents [9]. In the context of ArMs, this strategy has been most successfully applied to iron catalysis with heme proteins [5]. Spearheaded by Arnold and coworkers in 2013, who evolved cytochrome P450 variants to enantio- and diastereoselective enzymes for cyclopropanation of styrene $[13,16]$, an array of compelling studies emphasizing the plasticity of these proteins followed. This comprised development of biocatalysts for cyclopropanation [28] with complementary stereoselectivity [14] and trifluoromethyl substitution [15], for olefin aziridination [29], as well as for nitrene insertion to create C-N [30-33] and S-N [34, 35] bonds and carbene insertion into $\mathrm{N}-\mathrm{H}[36,37]$ and $\mathrm{S}-\mathrm{H}[38]$ bonds, to name but a few. Probably one of the most progressive recent studies is the repurposing of a cytochrome c variant from Rhodothermus marinus to form carbon-silicon bonds at high TONs and enantioselectivities [12]. Beyond the P450 domain other natural metalloenzymes have been repurposed, such as iron halogenase SyrB2 from Pseudomonas syringae B301D to catalyze azidation and nitration of non-activated aliphatic $\mathrm{C}-\mathrm{H}$ bonds [39] .

Unfortunately, for some chemically desirable transformations Nature does not (yet) provide promiscuous reactivity, which is essential for any directed evolution effort [9]. In these cases, rational protein design can offer valuable means to introduce entirely new reactivity into proteins, either by de novo design of synthetic (bottom-up) or based on existing protein folds (top-down). The group of Pecoraro, for instance, has designed a synthetic three-stranded coiled coil protein with a catalytically competent $\mathrm{Zn}(\mathrm{II})$ and a stabilizing $\mathrm{Hg}(\mathrm{II})$ center $[40,41]$. This protein exhibited hydrolytic activity for $p$ nitrophenyl acetate and $\mathrm{CO}_{2}$ hydration, the latter of which was later highly improved in another synthetic Zn-binding scaffold [42]. However, successful examples of strict de novo design of ArMs remain scarce [40], likely due to difficulties in designing stable folds with catalytic metal-binding sites from scratch. As an alternative, redesign of natural proteins to endow these with non-inherent catalytic activities was applied [17, 43]. In a seminal study Baker and coworkers applied computational design and directed evolution to create a highly active organophosphate hydrolase based on a 
mononuclear zinc deaminase, emphasizing the synergistic potential of these two methods [43].

A widely adopted and arguably pragmatic approach is the introduction of non-canonical catalytic metal(-complexes) (i.e. artificial cofactors) into proteins using appropriate anchoring strategies (vide infra). Synthetic heme derivatives have been used in which either iron is replaced by metals including $\mathrm{Mn}$, Co and Ir [11, 31, 44-46] or the structure of the porphyrin ligand is altered [44, 47]. Oohora et al. reconstituted myoglobin from horse heart with a Mn-porphycene cofactor to afford an ArM for the challenging hydroxylation of $\mathrm{C}\left(\mathrm{sp}^{3}\right)-\mathrm{H}$ bonds [44]. The group of Hartwig recently introduced Ircontaining heme into mutants of apo-myoglobin from Physeter macrocephalus creating ArMs for intramolecular $\mathrm{C}\left(\mathrm{sp}^{3}\right)-\mathrm{H}$ insertion of carbenes and intermolecular carbene addition to olefins, albeit at low activities [45]. This concept was later improved using a thermophilic protein variant and directed evolution to afford highly active variants for carbene insertion into $\mathrm{C}\left(\mathrm{sp}^{3}\right)-\mathrm{H}[46]$ and later extended to $\mathrm{C}-\mathrm{H}$ amination [11]. Likewise, artificial cofactors without natural equivalent have been used. These are fully synthetic metal complexes with a suitable anchoring moiety for the specific localization in the corresponding protein. Lewis and colleagues covalently anchored dirhodium complexes in the , -barrel protein tHisF [48] and later in a prolyl oligopeptidase [49], yielding ArMs for cyclopropanation and $\mathrm{Si}-\mathrm{H}$ bond insertion reactions. The latter host protein was evolved by iterative site-directed mutagenesis to a water-tolerant, enantioselective cyclopropanation enzyme [49]. Following pioneering works of Whitesides [4] several ArMs have been created relying on biotinylated transition metal cofactors and (strept-)avidin as the cognate host protein. Important recent reports exploiting this strategy are the creation of a rhodium ArM for asymmetric $\mathrm{C}-\mathrm{H}$ activation by Hyster et al. [50], which exhibited nearly 100 -fold rate acceleration compared to the free rhodium complex, as well as the combination of an iridium-based artificial transfer hydrogenase with several natural enzymes in one-pot reaction cascades by Köhler et al. [19]. Albeit in vitro, the latter represents an important step towards ArM application in complex reaction networks and artificial pathways. In vivo applications in mind, we have recently reported on the development and directed evolution of artificial metathases by combining a ruthenium-based cofactor with streptavidin in the periplasm of $E$. coli in aqueous medium under aerobic conditions [18]. Relying on the production of fluorescent umbelliferone by olefin metathesis, this enabled genetic optimization directly on whole cells without processing or purification of protein 
variants, thereby significantly increasing the throughput. The resulting metathases exhibited significantly improved activities for the screening substrate, albeit at low TONs, and for other di-olefin compounds.

Hence, the presented ensemble of recent developments in the ArM field (Table 1), while not comprehensive and likely subjective, emphasizes their potential for the creation of catalytic novelty in bio- and transition metal catalysis.

\section{Challenges for In Vivo Application of ArMs}

Despite significant recent advances, the in vivo implementation of ArMs imposes stringent challenges on chemists and metabolic engineers (Figure 2). Important obstacles include: i) choice of and expression strategy for the scaffold protein, ii) cellular uptake of metal cofactors, iii) intracellular ArM assembly, and iv) bioorthogonality of ArM (-reaction) and host cell (i.e. inhibition and cytotoxicity), which are individually discussed below.

$$
\text { are individually discussed below. }
$$

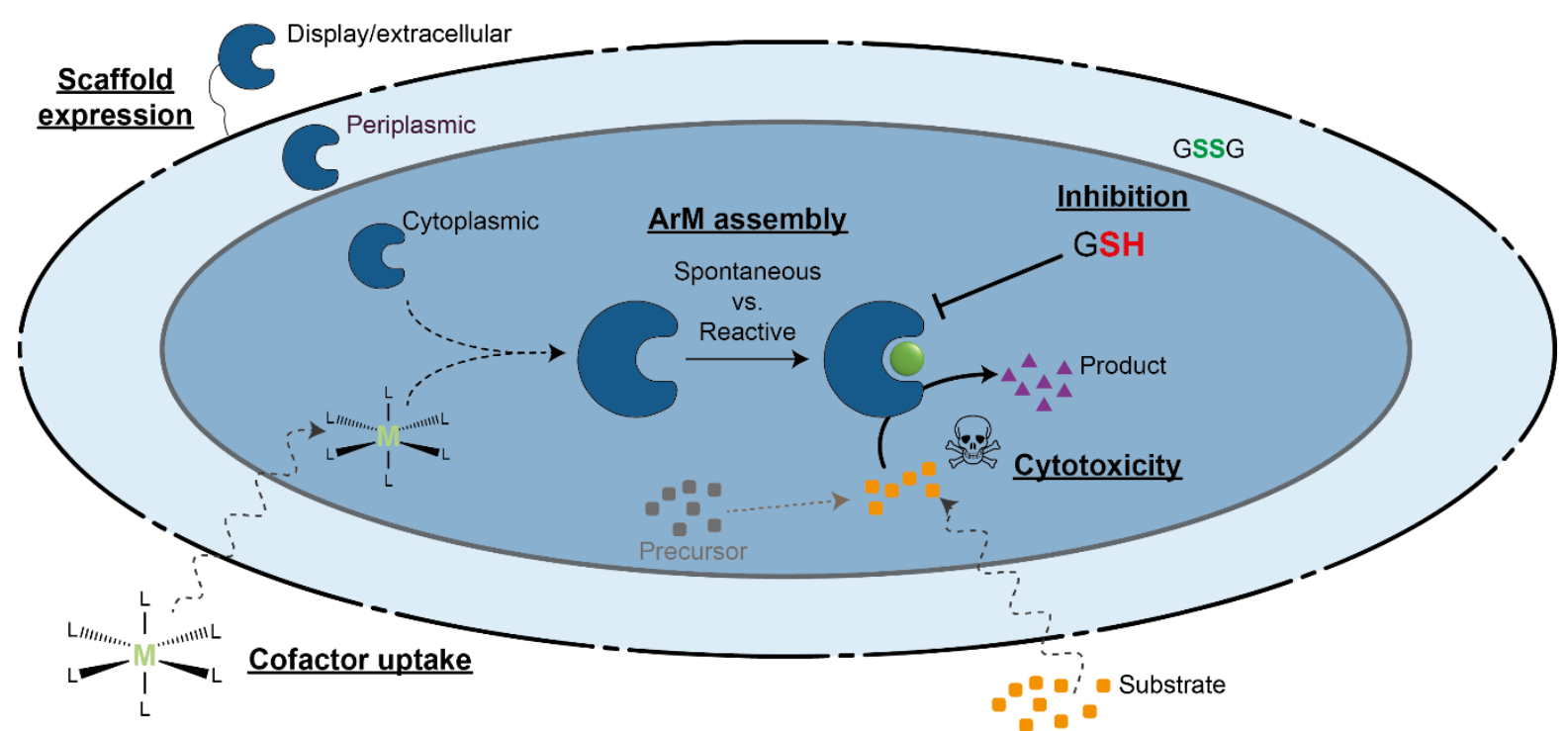

Figure 2. Critical challenges for in vivo implementation of ArMs. A number of obstacles need to be overcome in order to successfully implement ArMs in living cells. These include: i) the choice of the respective scaffold protein and appropriate ways for its expression, ii) cellular cofactor uptake and iii) subsequent assembly of the holoenzyme, as well as iv) considerations regarding the mutual interaction between the ArM and the host cell (i.e. inhibition and cytotoxicity). M: metal atom/ion, L: Ligand, GSH/GSSG: reduced/oxidized glutathione (disulfide). 
Table 1. Key features of selected recent studies on ArM development

\begin{tabular}{|c|c|c|c|c|c|}
\hline Cofactor $^{\mathrm{a}}$ & Protein ${ }^{a}$ & Anchoring & Reaction & $\begin{array}{l}\text { Whole Cell } \\
\text { Application }\end{array}$ & Refs \\
\hline \multicolumn{6}{|l|}{ Repurposing } \\
\hline \multirow{15}{*}{$\mathrm{Fe}(\mathrm{III})$, heme } & \multirow{9}{*}{$\begin{array}{l}\text { Cytochrome } \\
\text { P450/411 }\end{array}$} & \multirow{15}{*}{$\begin{array}{l}\text { Non- } \\
\text { covalent }\end{array}$} & Cyclopropanation of styrene & No & [13] \\
\hline & & & derivatives & Yes & [16] \\
\hline & & & Aziridination of aryl olefins & Yes & [29] \\
\hline & & & & Yes & [30] \\
\hline & & & $\mathrm{C}-\mathrm{H}$ amination (intramolecular) & No & [32] \\
\hline & & & $\mathrm{C}-\mathrm{H}$ amination (intermolecular) & Yes & [33] \\
\hline & & & & No & [34] \\
\hline & & & Sulfimidation & Yes & [35] \\
\hline & & & Carbene insertion into $\mathrm{N}-\mathrm{H}$ & Yes & [36] \\
\hline & $\begin{array}{l}\text { Rhodothermus } \\
\text { marinus } \\
\text { cytochrome c }\end{array}$ & & Carbene insertion into Si-H & Yes & [12] \\
\hline & \multirow{5}{*}{$\begin{array}{l}\text { Sperm whale } \\
\text { myoglobin }\end{array}$} & & $\begin{array}{l}\text { Cyclopropanation of styrene and } \\
\text { other aryl olefins }\end{array}$ & $\begin{array}{l}\text { No } \\
\text { Yes }\end{array}$ & $\begin{array}{l}{[28]} \\
{[14]}\end{array}$ \\
\hline & & & $\begin{array}{l}\text { Cyclopropanation of trifluoromethyl } \\
\text { substituted styrene and aryl olefins }\end{array}$ & Yes & [15] \\
\hline & & & Carbene insertion into $\mathrm{N}-\mathrm{H}$ & No & [37] \\
\hline & & & Carbene insertion into S-H & No & [38] \\
\hline & & & $\mathrm{C}-\mathrm{H}$ amination (intramolecular) & No & [31] \\
\hline $\begin{array}{l}\mathrm{Fe}(\mathrm{II}) \\
\text { haloferryl }\end{array}$ & $\begin{array}{l}\text { Pseudomonas } \\
\text { syringe B301D } \\
\text { halogenase SyrB2 }\end{array}$ & $\begin{array}{l}\text { Direct/ } \\
\text { dative }\end{array}$ & Azidation and nitration of $\mathrm{C}-\mathrm{H}$ & No & [39] \\
\hline \multicolumn{6}{|l|}{ (Re-)design } \\
\hline $\mathrm{Zn}(\mathrm{II})+\mathrm{Hg}(\mathrm{II})$ & $\begin{array}{l}\text { Synthetic triple } \\
\text { coiled coil }\end{array}$ & \multirow{4}{*}{$\begin{array}{l}\text { Direct/ } \\
\text { dative }\end{array}$} & $\begin{array}{l}\text { Hydrolysis of p-nitrophenyl acetate } \\
\text { and } \mathrm{CO}_{2} \text { hydration }\end{array}$ & No & [40] \\
\hline \multirow{3}{*}{$\mathrm{Zn}(\mathrm{II})$} & $\begin{array}{l}\text { Synthetic triple } \\
\text { coiled coil }\end{array}$ & & $\mathrm{CO}_{2}$ hydration & No & [42] \\
\hline & $\begin{array}{l}\text { Mouse adenosine } \\
\text { deaminase }\end{array}$ & & Organophosphate hydrolysis & No & [43] \\
\hline & Cytochrome cb $_{562}$ & & $\beta$-lactam hydrolysis & Yes & {$[17]$} \\
\hline \multicolumn{6}{|c|}{ Artificial cofactors } \\
\hline $\begin{array}{l}\text { Mn(III), } \\
\text { porphycene }\end{array}$ & $\begin{array}{l}\text { Horse heart } \\
\text { myoglobin }\end{array}$ & \multirow{4}{*}{$\begin{array}{l}\text { Non- } \\
\text { covalent }\end{array}$} & Hydroxylation of $\mathrm{C}-\mathrm{H}$ & No & [44] \\
\hline \multirow{3}{*}{$\begin{array}{l}\operatorname{Ir}(I I I)-(\mathrm{Me}) \\
\text { heme }\end{array}$} & $\begin{array}{l}\text { Physeter } \\
\text { macrocephalus } \\
\text { myoglobin }\end{array}$ & & $\begin{array}{l}\text { Carbene insertion into } \mathrm{C}-\mathrm{H} \text { and } \\
\text { carbene olefin addition }\end{array}$ & No & [45] \\
\hline & \multirow{2}{*}{$\begin{array}{l}\text { Sulfolobus } \\
\text { solfataricus P450 } \\
\text { CYP119 }\end{array}$} & & Carbene insertion into $\mathrm{C}-\mathrm{H}$ & No & [46] \\
\hline & & & C-H amination (intramolecular) & No & [11] \\
\hline \multirow{2}{*}{$\begin{array}{l}\text { di-Rh(II), tetra- } \\
\text { carboxylate } \\
\text { complex }\end{array}$} & $\begin{array}{l}\text { Thermotoga } \\
\text { maritima tHisF }\end{array}$ & \multirow{2}{*}{ covalent } & $\begin{array}{l}\text { Cyclopropanation of styrene } \\
\text { derivatives and carbene insertion } \\
\text { into Si-H }\end{array}$ & No & [48] \\
\hline & $\begin{array}{l}\text { Pyrococcus } \\
\text { furiosus prolyl } \\
\text { oligopeptidase }\end{array}$ & & $\begin{array}{l}\text { Cyclopropanation of styrene } \\
\text { derivatives }\end{array}$ & No & [49] \\
\hline $\begin{array}{l}\mathrm{Rh}(\mathrm{III}), \mathrm{Cp}^{*}- \\
\text { biotin }\end{array}$ & \multirow{3}{*}{ Streptavidin } & \multirow{3}{*}{$\begin{array}{l}\text { Non- } \\
\text { covalent / } \\
\text { supra- } \\
\text { molecular }\end{array}$} & Asymmetric $\mathrm{C}-\mathrm{H}$ activation & No & [50] \\
\hline $\begin{array}{l}\text { Ir(III), } \mathrm{Cp}^{*}- \\
\text { biotin }\end{array}$ & & & $\begin{array}{l}\text { Artificial transfer hydrogenation } \\
\text { (incl. cascades) }\end{array}$ & No & [19] \\
\hline $\begin{array}{l}\text { Ru(II), } \\
\text { Hoveyda- } \\
\text { Grubbs-biotin }\end{array}$ & & & Olefin metathesis & Yes & [18] \\
\hline
\end{tabular}

a in cases with multiple metals or screened proteins the most active or evolved ones are given 
In principle, ArMs can be created from any scaffold protein into which the desired metal(-complex) can be anchored. Accordingly, several proteins from various host organisms have been used [5, 27]. In addition to practical requirements such as the ability to synthesize the protein in sufficiently high amounts (e.g. in E. coli), further considerations for scaffold selection apply for ArMs [6].

Stability under the required reaction conditions and evolvability are important requirements [6]. Therefore, proteins from thermophilic organisms are frequently selected as starting points for directed evolution campaigns because of their highly stable folds and tolerance to mutation $[51,52]$. ArMs have recently been created from thermostable variants of a synthase from histidine biosynthesis [48, 53], a prolyl oligopeptidase [49], P450 cytochromes [11, 12, 46], and a cupin-like protein [54]. Other specifications can restrict the protein repertory further. Repurposing approaches, for instance, rely on intrinsic side reactivities, which sometimes requires screening to identify a suitable origin for directed evolution [12, 13]. Likewise, metal cofactor anchoring (vide infra) can limit the available range of candidate proteins significantly if inherent metal binding or affinity to supramolecular anchoring moieties is required. Once selected, the protein can be expressed in a desired host organism, which in the field of ArMs has thus far largely been performed in $E$. coli due to ease of cultivation and availability of versatile methods for genetic engineering. "Traditional" cytosolic expression is commonly used before purification using standard procedures (e.g. affinity chromatography). While useful to isolate preparative protein quantities, this strategy is not necessarily the best choice for whole cell and in vivo applications. In particular restricted cofactor uptake and inhibition of transition metal catalysis by cytosolic compounds may speak in favor of alternative production pathways like periplasmic or extracellular expression (vide infra). The former was successfully used by Song and Tezcan to create an artificial metallo- $\beta$-lactamase [17] and later by us to implement and evolve ArMs for olefin metathesis in E. coli [18].

Although thus far largely under-appreciated in this specific context, other organisms may prove valuable, for instance for preparative ArM applications. The methylotrophic yeast Pichia pastoris, for example, is a potent, well-characterized host for high-yield secretory protein production, offers high solvent tolerance and access to cheap carbon and energy sources [55], and has been recently used for the production of a 
streptavidin-based artificial imine reductase [56]. Similarly, other hosts including mammalian cells could facilitate future ArM development in vivo.

\section{Cofactor Uptake}

Another important aspect is cellular uptake of cofactors as a limitation for ArM usage in vivo in contrast to in vitro scenarios where the scaffold protein is freely accessible. In particular artificial cofactors with complex ligands frequently exceed the molecular weight exclusion cut-off of outer membrane porins ( 600 Da) [57] and do not have access to a specific cellular uptake machinery like natural cofactors such as heme [58]. During our aforementioned study on artificial metathases, we identified the uptake of the ruthenium cofactor as a major bottleneck [18]. Although in vivo assembly and directed evolution was still feasible in spite of prevailing cofactor uptake limitations by adding surplus cofactor to the cells and subsequently eliminating excess by washing, a restricted cofactor uptake imposes major limitations for preparative whole cell and in vivo applications, since it reduces overall yield of the ArM reaction. Similar arguments may be made for non-permeable reaction substrates.

In principle, uptake limitations can be overcome by different measures on both the chemical and biological side. They should be considered during initial cofactor design, and size reduction as well as chemical modification [59] are measures with potential to improve uptake. On the biological side, the scaffold protein can be expressed in the periplasm or on the cell surface to avoid requirement for cofactor transit through membranes (vide supra) [60]. Overexpression of suitable outer membrane transport proteins was shown to improve uptake of metal-substituted porphyrin derivatives [31, $47,61]$ and engineering of pore proteins may help to elevate the cut-off of the outer membrane [62]. Alternatively, the permeability of the cell envelope can be increased by chemical treatment. For $E$. coli we have observed improvement of cofactor uptake in presence of high salt concentrations, which are known to facilitate uptake of large compounds [63]. Wallace and Balskus suggested micelles to enhance cyclopropanation of styrene produced in situ by $E$. coli and suggest increased membrane permeability as contributing factor for the observed improvement [64].

\section{Cofactor anchoring}

Quantitative and precise localization of metal cofactors within protein scaffolds is an important prerequisite to create functional and evolvable metalloenzymes. To this end, 
several strategies have been pursued, which have been thoroughly reviewed previously (e.g. [3, 5, 6, 27]). While all of these strategies have successfully been applied in ArM assembly, they arguably differ significantly in view of their utility for whole cell and in vivo applications [60]. In this context, two main coupling modes, reactive covalent and spontaneous noncovalent coupling, can be distinguished.

\section{Reactive Coupling}

Covalent cofactor attachment to amino acid residues ensures stable anchoring and allows for a high degree of flexibility with respect to scaffold protein choice and metal positioning [5]. However, natural conjugative residues (lysine, cysteine) lack specificity in scenarios with multitudes of non-target proteins present in the reaction mixture where off-target binding is likely. To achieve bioorthogonality site-specific introduction of non-canonical residues by amber stop codon suppression can be used [65]. The group of Lewis introduced p-azido-L-phenylalanine within the pore of the , -barrel protein tHisF [48] and later within a prolyl oligopeptidase [49] to conjugate different artificial cofactors by copper-free click chemistry. An alternative way to achieve higher specificity is the exploitation of active site residues for bioconjugation [5]. Eppinger demonstrated the coupling of rhodium and ruthenium half-sandwich complexes to the nucleophilic active site cysteine of papain relying on inhibitors for this protein to ensure both efficient bond formation via a reactive epoxide moiety and precise positioning by non-covalent interaction [66]. This allowed for ArM assembly at substoichiometric cofactor-to-protein ratios and the creation of an enantioselective hydrogenation biocatalyst from achiral metal complexes.

However, covalent ArM assembly in complex biological systems remains challenging. Major obstacles include limitations in biocompatibility (toxicity) and bioorthogonality (cross-reactivity), inefficiency of non-canonical residue introduction, and poor efficiency of the coupling reaction. The latter imposes the use of multiple cofactor equivalents to achieve quantitative protein conjugation [60].

\section{Spontaneous Non-Covalent Coupling}

A conceptually different approach builds on anchoring of the metal(-complex) via noncovalent interactions. In analogy to many natural metalloenzymes, efforts for ArM creation have been reported that rely on the assembly of active sites by direct interaction of the metal with coordinating residues such as histidine [5, 27]. In this case 
the metal's first ligand sphere is partially or fully completed by the protein and assembly occurs spontaneously, rendering complicated and potentially detrimental reactive steps dispensable. To this end, ArMs can be created by re-purposing of natural metalbinding sites, either building on catalytic promiscuity of the native metal or by reconstitution with nonnative metals. Particularly noteworthy studies include the computational redesign and directed evolution of a zinc deaminase to an organophosphate hydrolase [43] and the reconstitution of a manganese-binding protein from the cupin family with osmium( $\mathrm{VI})$ resulting in a thermostable artificial peroxygenase with high TON [54]. Alternatively, metal centers can be created de novo based on existing or fully synthetic protein folds [17, 40, 42, 67]. Based on earlier works of Lee and Schultz [68], the group of Roelfes used amber suppression to introduce the non-canonical amino acid (2,2'-bipyridine-5yl)alanine into the transcription factor $\mathrm{LmrR}$ in vivo thereby creating an ArM for asymmetric Friedel-Crafts alkylation [69]. This bidentate ligand allows for straightforward site-specific introduction of metal-chelating capacity simplifying active site creation by dative interaction.

The natural prosthetic group heme as well as synthetic derivatives thereof have been extensively studied in the context of enzyme re-purposing and ArM creation (for comprehensive reviews please refer to $[3,7,27])$. Arguably, natural heme proteins and enzymes exhibit high affinity and specificity for the porphyrin scaffold, which renders holoenzyme assembly comparably simple. This has been exploited for ArM creation relying either on catalytic promiscuity of natural iron cofactor [12-16, 28, 29, 31, 33, 38] or by introducing synthetic derivatives of the latter [11, 31, 44-47]. Moreover, the available natural transport machinery for heme may be exploited to enhance cofactor uptake (vide supra) [47]. Importantly, reconstitution of proteins with non-native metals requires either ability to directly express the apoprotein or to retrieve it by removal of bound metal ex post (e.g. by dialysis) [5]. This premise, which stems from the lower affinity of non-native versus native metal, imposes additional challenges on the in vivo assembly of these ArMs. To overcome this limitation, Brustad and colleagues evolved a cytochrome P450 variant that selectively binds non-proteinogenic iron deuteroporphyrin IX in vivo over endogenous heme, thereby creating an orthogonal enzyme-cofactor pair [47].

Lastly, introduction of catalytic metals into proteins has been achieved via high-affinity protein-ligand interaction frequently referred to as supramolecular or "Trojan horse" strategy [5]. Although this approach limits the scope of target proteins to those that 
exhibit sufficiently strong interaction with suitable anchoring moieties, it has several compelling assets for in vivo applications [60]: First, assembly occurs spontaneously upon mixing of scaffold protein and cofactor in solution without reactive coupling or prior binding site design and optimization. Second, metal anchoring is specific and essentially quantitative even at equimolar protein-cofactor ratios (provided sufficient affinity). And third, modular separation of catalytic and anchoring moiety allows for facile exchange of host proteins for given catalysts and similarly swapping of catalytic functionality in the same scaffold. Amongst supramolecular approaches the (strept)avidin-biotin technology is likely the most widespread and versatile one. This may be traced back to: i) the nearly irreversible biotin binding $\left(K_{D} \sim 10^{-14} \mathrm{M}\right)$, which is exploited in several applications outside the domain of ArMs and ii) high chemical and physical stability of (strept-)avidin. These properties have led to a diverse array of ArMs with several catalytic metals and target reactions, which has been reviewed elsewhere (e.g. $[3,5])$. Furthermore, other protein-ligand pairs have been used for ArM construction including carbonic anhydrase and cognate sulfonamide inhibitors [70, 71], xylanase with carboxylated porphyrin derivatives [72, 73], and $\beta$-lactoglobulin and aliphatic chains [74].

\section{Inhibition and Toxicity}

In contrast to defined in vitro scenarios, ArM application in vivo requires consideration of mutual interactions between the (ideally bio-orthogonal) ArM and the host. This comprises inhibition of catalysis by cellular components as well as toxicity of the ArM (reaction) against the host cell. Glutathione has been identified as a major inhibitor of transition metal reactions in cell lysates $[18,75]$, likely due to formation of metal-thiolate complexes, and other cell-derived agents such as proteins, nucleic acids and reactive or chelating metabolites come to mind as potential poisons. While shielding of the cofactor by the protein can enhance stability [18, 49]), additional measures are required to avoid the said limitations. Quenchers can diminish inhibition, which has been capitalized on using diamide to oxidize thiols in cell lysates in ArM-catalyzed asymmetric transfer hydrogenation [75] or by application of reducing agents (e.g. sodium dithionite) and anaerobic conditions for oxygen-sensitive reactions $[12,13,16$, $28,38]$. However, biocompatibility of the quencher has to be taken into account for in vivo applications. Alternatively, placing the ArM in another compartment, the periplasm, whose oxidative environment lacks large amounts of free thiols and other 
potentially detrimental agents from the cytosol, has been succesfully used for ArM development $[17,18,60]$.

Besides catalyst poisoning, cytotoxicity is a major barrier hindering in vivo applicability of many ArM reactions. It can be caused by the ArM as such, by substrates, intermediates and products of the corresponding reaction, or by additives. Notably, some recent works in the ArM field involve biotransformations with whole cells of E. coli, which has the potential to simplify production and represents a first step towards in vivo utilization. However, most of these studies apply conditions limiting their in vivo utility including the presence of significant amounts of cytotoxic agents such as organic solvents, styrene (derivatives), diazo compounds, and azides as well as strict anaerobic conditions enforced by oxygen stripping and reductants.

Use of biocompatible solvents such as dimethyl sulfoxide, stepwise substrate addition, and in situ product removal can help to mitigate toxic effects. To this end, vitamin Ederived micelles were shown to elevate styrene production in $E$. coli beyond toxicity limits, which was exploited for iron-catalyzed in situ cyclopropanation (no ArM) under aerobic conditions $[64,76]$. The group of Fasan recently reported on a two-vessel setup for ex situ generation of highly toxic and volatile 2-diazo-1,1,1-trifluoroethane, which was used as carbene donor in myoglobin-catalyzed cyclopropanation of styrene derivatives using $E$. coli cells [15].

Importantly, due to a plethora of potential contributors to the global phenomenon of cytotoxicity, it is arguably difficult to address in a generic manner, and careful evaluation is necessary for individual ArMs.

\section{Concluding Remarks and Future Perspectives}

As highlighted in this synopsis, ArMs constitute a promising technology merging crucial assets of transition metal catalysis and enzymology, which can be exploited to create new biocatalysts for organic synthesis and to expand Nature's enzymatic arsenal. From the chemist's viewpoint, this concept can be readily applied for transformations of ever increasing intricacy and with efficiencies approaching economic viability. On the contrary, the synthetic biology angle has hitherto been largely underappreciated, very likely due to limitations of ArM applicability in living cells.

Full integration of ArMs into metabolic networks of cells, however, holds great promises for future applications. Firstly, it will allow to apply the entire potential of laboratory 
evolution to these new biocatalysts, which will dramatically increase pace and

421 throughput of optimization and enable the development of ArMs with highly improved

422

423

424 and entirely novel catalytic properties. To this end, ArMs could be subjected to highthroughput screening assays without the need for extensive processing procedures [18]. Moreover, Darwinian selection schemes, in which ArM reactions are causally coupled to the survival or proliferation of the host organism, could be applied, which enables retrieval of improved variants from extremely large pools via competitive onepot growth experiments. Second, in vivo integration of new reactivities by ArMs (and other artificial enzymes) will eventually allow for implementation of novel metabolic routes for sustainable production of previously inaccessible chemicals from renewable feedstocks [77-79]. Lastly, beyond the aforementioned synthetic applications, transition metal catalysis and consequently ArMs could be used for biochemical and medical applications, which was not outlined herein but elaborated on elsewhere (e.g. $[24,25,80])$.

In quintessence, the assimilation of ArMs by living cells is a highly auspicious, yet challenging, endeavor and may contribute to a future "fourth wave" of biocatalysis following Bornscheuer's metaphor [1].

\section{<Outstanding Questions Box>}

What are the most pressing chemical challenges that ArMs could solve?

Will ArMs be able to make it "out of the niche" by outcompeting small molecule catalysts in large scales and for wide applications in the near future and can they contribute to the transition towards a sustainable, bio-based economy?

Can we combine multiple ArMs and natural enzymes with each other to engineer entire artificial pathways in living organisms, which lead to the production of previously inaccessible bio-products and what will the latter look like?

Is it possible to systematically install functional ArMs in living organisms that are propagating in the presence of the ArM reaction or even benefit from it and what is the potential of bringing non-natural metals (e.g. iridium, ruthenium, rhodium, palladium, gold, osmium etc.) into synthetic biology?

Will we be able to establish biosynthesis of non-canonical cofactors to render their addition to the cells obsolete?

What is the potential of in vivo utilization of ArMs beyond bio-production and preparative chemistry? <।Outstanding Questions Box> 


\section{References}

4551 Bornscheuer, U.T. et al. (2012) Engineering the third wave of biocatalysis. Nature 485, 185-194

4562 Bornscheuer, U.T. (2016) Chemical biology: A radical change in enzyme catalysis. Nature 540, 345-

$457 \quad 346$

4583 Hyster, T.K. and Ward, T.R. (2016) Genetic optimization of metalloenzymes: enhancing enzymes for 459 non-natural reactions. Angew. Chem. Int. Ed. Engl. 55, 7344-7357

4604 Wilson, M.E. and Whitesides, G.M. (1978) Conversion of a protein to a homogeneous asymmetric 461 hydrogenation catalyst by site-specific modification with a diphosphinerhodium(I) moiety. J. Am.

462 Chem. Soc. 100, 306-307

4635 Lewis, J.C. (2013) Artificial metalloenzymes and metallopeptide catalysts for organic synthesis. ACS

464 Catal. 3, 2954-2975

4656 llie, A. and Reetz, M.T. (2015) Directed evolution of artificial metalloenzymes. Isr. J. Chem. 55, 51$466 \quad 60$

4677 Renata, H. et al. (2015) Expanding the enzyme universe: accessing non-natural reactions by

468 mechanism-guided directed evolution. Angew. Chem. Int. Ed. Engl. 54, 3351-3367

4698 Upp, D.M. and Lewis, J.C. (2017) Selective C-H bond functionalization using repurposed or artificial 470 metalloenzymes. Curr. Opin. Chem. Biol. 37, 48-55

4719 Hammer, S.C. et al. (2017) Design and evolution of enzymes for non-natural chemistry. Curr. Opin.

472 Green Sust. Chem. 7, 23-30

47310 Obexer, R. et al. (2017) Emergence of a catalytic tetrad during evolution of a highly active artificial aldolase. Nat. Chem. 9, 50-56

11 Dydio, P. et al. (2017) Chemoselective, enzymatic C-H bond amination catalyzed by a cytochrome P450 containing an $\operatorname{Ir}(\mathrm{Me})$-PIX cofactor. J. Am. Chem. Soc. 139, 1750-1753

12 Kan, S.B. et al. (2016) Directed evolution of cytochrome c for carbon-silicon bond formation: bringing silicon to life. Science 354, 1048-1051

47913 Coelho, P.S. et al. (2013) Olefinic cyclopropanation via carbene transfer catalyzed by engineered 480 cytochrome P450 enzymes. Science 339, 307-310

48114 Bajaj, P. et al. (2016) Gram-scale synthesis of chiral cyclopropane-containing drugs and drug

482 precursors with engineered myoglobin catalysts featuring complementary stereoselectivity. Angew.

483 Chem. Int. Ed. Engl. 55, 16110-16114

48415 Tinoco, A. et al. (2017) Highly diastereo- and enantioselective synthesis of trifluoromethyl-

485 substituted cyclopropanes via myoglobin-catalyzed transfer of trifluoromethylcarbene. J. Am. Chem.

486 Soc. 139, 5293-5296

48716 Coelho, P.S. et al. (2013) A serine-substituted P450 catalyzes highly efficient carbene transfer to olefins in vivo. Nat. Chem. Biol. 9, 485-487

17 Song, W.J. and Tezcan, F.A. (2014) A designed supramolecular protein assembly with in vivo enzymatic activity. Science 346, 1525-1528

18 Jeschek, M. et al. (2016) Directed evolution of artificial metalloenzymes for in vivo metathesis. Nature 537, 661-665

19 Kohler, V. et al. (2013) Synthetic cascades are enabled by combining biocatalysts with artificial metalloenzymes. Nat. Chem. 5, 93-99

20 Okamoto, Y. et al. (2016) An NAD(P)H-dependent artificial transfer hydrogenase for multienzymatic cascades. J. Am. Chem. Soc. 138, 5781-5784

$21 \mathrm{Liu}$, Z. et al. (2016) Upregulation of an artificial zymogen by proteolysis. Angew. Chem. Int. Ed. Engl. 55, 11587-11590

22 Bersellini, M. and Roelfes, G. (2017) A metal ion regulated artificial metalloenzyme. Dalton Trans. $46,4325-4330$

23 Okamoto, Y. and Ward, T.R. (2017) Cross-regulation of an artificial metalloenzyme. Angew. Chem. Int. Ed. Engl. 56, 1-6

24 Sasmal, P.K. et al. (2013) Metal complex catalysis in living biological systems. Chem. Commun. 49, 1581-1587 
25 Volker, T. et al. (2014) Progress towards bioorthogonal catalysis with organometallic compounds. Angew. Chem. Int. Ed. Engl. 53, 10536-10540

26 Ball, Z.T. (2013) Designing enzyme-like catalysts: a rhodium(II) metallopeptide case study. Acc. Chem. Res. 46, 560-570 27 Yu, F.T. et al. (2014) Protein design: toward functional metalloenzymes. Chem. Rev. 114, 34953578

28 Bordeaux, M. et al. (2015) Highly diastereoselective and enantioselective olefin cyclopropanation using engineered myoglobin-based catalysts. Angew. Chem. Int. Edit. Engl. 54, 1744-1748 29 Farwell, C.C. et al. (2015) Enantioselective enzyme-catalyzed aziridination enabled by active-site evolution of a cytochrome P450. ACS Central Sci. 1, 89-93 30 McIntosh, J.A. et al. (2013) Enantioselective intramolecular C-H amination catalyzed by engineered cytochrome P450 enzymes in vitro and in vivo. Angew. Chem. Int. Ed. Engl. 52, 9309-9312 31 Bordeaux, M. et al. (2014) Intramolecular C(sp(3))-H amination of arylsulfonyl azides with engineered and artificial myoglobin-based catalysts. Bioorgan. Med. Chem. 22, 5697-5704 32 Hyster, T.K. et al. (2014) Enzyme-controlled nitrogen-atom transfer enables regiodivergent C-H amination. J. Am. Chem. Soc. 136, 15505-15508

33 Prier, C.K. et al. (2017) Enantioselective, intermolecular benzylic C-H amination catalysed by an engineered iron-haem enzyme. Nat. Chem. 9, 629-634 34 Farwell, C.C. et al. (2014) Enantioselective imidation of sulfides via enzyme-catalyzed intermolecular nitrogen-atom transfer. J. Am. Chem. Soc. 136, 8766-8771 35 Prier, C.K. et al. (2016) Asymmetric enzymatic synthesis of allylic amines: a sigmatropic rearrangement strategy. Angew. Chem. Int. Ed. Engl. 55, 4711-4715 36 Wang, Z.J. et al. (2014) Cytochrome P450-catalyzed insertion of carbenoids into N-H bonds. Chem. Sci. 5, 598-601

37 Sreenilayam, G. and Fasan, R. (2015) Myoglobin-catalyzed intermolecular carbene N-H insertion with arylamine substrates. Chem. Commun. 51, 1744-1744

38 Tyagi, V. et al. (2015) Intermolecular carbene S-H insertion catalysed by engineered myoglobinbased catalysts. Chem. Sci. 6, 2488-2494

39 Matthews, M.L. et al. (2014) Direct nitration and azidation of aliphatic carbons by an irondependent halogenase. Nat. Chem. Biol. 10, 209-215

40 Zastrow, M.L. et al. (2012) Hydrolytic catalysis and structural stabilization in a designed metalloprotein. Nat. Chem. 4, 118-123

41 Dieckmann, G.R. et al. (1997) De novo design of mercury-binding two- and three-helical bundles. J. Am. Chem. Soc. 119, 6195-6196

42 Cangelosi, V.M. et al. (2014) A de novo designed metalloenzyme for the hydration of $\mathrm{CO}_{2}$. Angew. Chem. Int. Ed. Engl. 53, 7900-7903

43 Khare, S.D. et al. (2012) Computational redesign of a mononuclear zinc metalloenzyme for organophosphate hydrolysis. Nat. Chem. Biol. 8, 294-300

44 Oohora, K. et al. (2013) C(sp(3))-H bond hydroxylation catalyzed by myoglobin reconstituted with manganese porphycene. J. Am. Chem. Soc. 135, 17282-17285

45 Key, H.M. et al. (2016) Abiological catalysis by artificial haem proteins containing noble metals in place of iron. Nature 534, 534-537.

46 Dydio, P. et al. (2016) An artificial metalloenzyme with the kinetics of native enzymes. Science 354, 102-106

47 Reynolds, E.W. et al. (2016) An evolved orthogonal enzyme/cofactor pair. J. Am. Chem. Soc. 138, 12451-12458

48 Yang, H. et al. (2014) A general method for artificial metalloenzyme formation through strainpromoted azide-alkyne cycloaddition. Chembiochem 15, 223-227

49 Srivastava, P. et al. (2015) Engineering a dirhodium artificial metalloenzyme for selective olefin cyclopropanation. Nat. Commun. 6, 7789

50 Hyster, T.K. et al. (2012) Biotinylated Rh(III) complexes in engineered streptavidin for accelerated asymmetric $\mathrm{C}-\mathrm{H}$ activation. Science $338,500-503$ 
51 Besenmatter, W. et al. (2007) Relative tolerance of mesostable and thermostable protein homologs to extensive mutation. Proteins Struct. Funct. Bioinf. 66, 500-506 52 Tokuriki, N. and Tawfik D.S. (2009) Stability effects of mutations and protein evolvability. Curr. Opin. Struc. Biol. 19, 596-604

53 Reetz, M.T. et al. (2008) A robust protein host for anchoring chelating ligands and organocatalysts. Chembiochem 9, 552-564

54 Fujieda, N. et al. (2017) A well-defined osmium-cupin complex: hyperstable artificial osmium peroxygenase. J. Am. Chem. Soc. 139, 5149-5155

55 Cereghino, J.L. and Cregg, J.M. (2000) Heterologous protein expression in the methylotrophic yeast Pichia pastoris. FEMS Microbiol. Rev. 24, 45-66

56 Nogueira, E.S. et al. (2014) High-level secretion of recombinant full-length streptavidin in Pichia pastoris and its application to enantioselective catalysis. Protein Expression Purif. 93, 54-62 57 Delcour, A.H. (2009) Outer membrane permeability and antibiotic resistance. Biochim. Biophys. Acta, Proteins Proteomics 1794, 808-816

58 Tong, Y. and Guo, M. (2009) Bacterial heme-transport proteins and their heme-coordination modes. Arch. Biochem. Biophys. 481, 1-15

59 Richter, M.F. et al. (2017) Predictive compound accumulation rules yield a broad - spectrum antibiotic. Nature 545, 299-304

60 Jeschek, M. et al. (2016) Periplasmic screening for artificial metalloenzymes. Methods Enzymol. 580, 539-556

61 Lelyveld, V.S. et al. (2011) Metal-substituted protein MRI contrast agents engineered for enhanced relaxivity and ligand sensitivity. J. Am. Chem. Soc. 133, 649-651

62 Krewinkel, M. et al. (2011) Engineering of an E. coli outer membrane protein FhuA with increased channel diameter. J. Nanobiotechnol. 9

63 Chen, G. et al. (2001) Isolation of high-affinity ligand-binding proteins by periplasmic expression with cytometric screening (PECS). Nat. Biotechnol. 19, 537-542

64 Wallace, S. and Balskus, E.P. (2016) Designer micelles accelerate flux through engineered metabolism in E. coli and support biocompatible chemistry. Angew. Chem. Int. Edit. Engl. 55, 60236027

65 Wang, L. et al. (2001) Expanding the genetic code of Escherichia coli. Science 292, 498-500

66 Reiner, T. et al. (2013) Metal-conjugated affinity labels: a new concept to create enantioselective artificial metalloenzymes. ChemistryOpen 2, 50-54

67 Podtetenieff, J. et al. (2010) An artificial metalloenzyme: creation of a designed copper binding site in a thermostable protein. Angew. Chem. Int. Ed. Engl. 49, 5151-5155

68 Lee, H.S. and Schultz, P.G. (2008) Biosynthesis of a site-specific DNA cleaving protein. J. Am. Chem. Soc. $130,13194-13195$

69 Drienovska, I. et al. (2015) Novel artificial metalloenzymes by in vivo incorporation of metalbinding unnatural amino acids. Chem. Sci. 6, 770-776

70 Monnard, F.W. et al. (2011) Human carbonic anhydrase II as a host for piano-stool complexes bearing a sulfonamide anchor. Chem. Commun. 47, 8238-8240

71 Zhao, J.M. et al. (2015) Carbonic anhydrase II as host protein for the creation of a biocompatible artificial metathesase. Org. Biomol. Chem. 13, 5652-5655

72 Ricoux, R. et al. (2008) Hemozymes peroxidase activity of artificial hemoproteins constructed from the Streptomyces lividans xylanase A and iron(III)-carboxy-substituted porphyrins. Bioconjugate Chem. 19, 899-910

73 Allard, M. et al. (2012) Incorporation of manganese complexes into xylanase: new artificial metalloenzymes for enantioselective epoxidation. Chembiochem 13, 240-251

74 Chevalley, A. et al. (2014) Artificial metalloenzymes derived from bovine beta-lactoglobulin for the asymmetric transfer hydrogenation of an aryl ketone - synthesis, characterization and catalytic activity. Dalton Trans. 43, 5482-5489 75 Wilson, Y.M. et al. (2014) Neutralizing the detrimental effect of glutathione on precious metal catalysts. J. Am. Chem. Soc. 136, 8928-8932 
60976 Wallace, S. and Balskus, E.P. (2015) Interfacing microbial styrene production with a biocompatible 610 cyclopropanation reaction. Angew. Chem. Int. Edit. Engl. 54, 7106-7109

61177 Otte, K.B. and Hauer, B. (2015) Enzyme engineering in the context of novel pathways and 612 products. Curr. Opin. Biotechnol. 35, 16-22

$61378 \mathrm{Erb}$, T.J. et al. (2017) Synthetic metabolism: metabolic engineering meets enzyme design. Curr.

614 Opin. Chem. Biol. 37, 56-62

61579 Hepworth, L.J. et al. (2017) Enzyme cascades in whole cells for the synthesis of chiral cyclic 616 amines. ACS Catal. 7, 2920-2925

61780 Tsubokura, K. et al. (2017) In vivo gold complex catalysis within live mice. Angew. Chem. Int. Edit. 618 Engl. 56, 3579-3584

619

620 